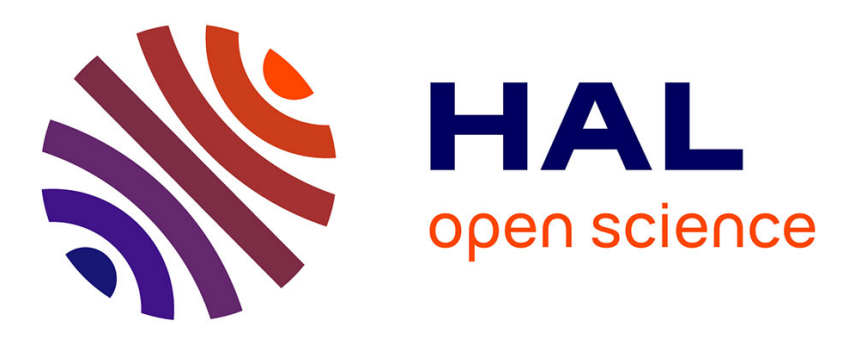

\title{
Method for measuring small optical absorption coefficients with use of a Shack-Hartmann wave-front detector
}

Sanichiro Yoshida, David H. Reitze, David B. Tanner, Justin D. Mansell

\section{- To cite this version:}

Sanichiro Yoshida, David H. Reitze, David B. Tanner, Justin D. Mansell. Method for measuring small optical absorption coefficients with use of a Shack-Hartmann wave-front detector. Applied optics, 2003, 42 (24), pp.4835-4840. hal-01092277

\section{HAL Id: hal-01092277 \\ https://hal.science/hal-01092277}

Submitted on 8 Dec 2014

HAL is a multi-disciplinary open access archive for the deposit and dissemination of scientific research documents, whether they are published or not. The documents may come from teaching and research institutions in France or abroad, or from public or private research centers.
L'archive ouverte pluridisciplinaire HAL, est destinée au dépôt et à la diffusion de documents scientifiques de niveau recherche, publiés ou non, émanant des établissements d'enseignement et de recherche français ou étrangers, des laboratoires publics ou privés. 


\title{
Method for measuring very small optical ab- sorption coefficients using a Shack-Hartmann wavefront detector
}

\author{
Sanichiro Yoshida \\ LIGO (Laser Interferometer Gravitational-wave Observatory) Livingston \\ Observatory \\ 19100 Ligo Lane, PO Box 940 Livingston, LA 70754 USA \\ Department of Chemistry and Physics \\ Southeastern Lousiana University, SLU 10878 Hammond, LA 70433 USA
}

\author{
David H. Reitze and David B. Tanner \\ Physics Department, University of Florida \\ P. O. Box 18440, Gainesville, FL 32611 USA
}

Justin D. Mansell

Intellite, Inc.

1717 Louisiana, Ste. 202 NE Albuquerque, NM 87110 USA

\begin{abstract}
We present a method for measuring absorption at the $1 \times 10^{-5} \mathrm{~cm}^{-1}$ level in high-quality optical materials. Using a Shack-Hartmann wavefront detector, thermal lensing in these materials may be measured. Then, the absorption coefficient may be estimated by fitting the observed deformation to a thermal lensing model based on the temperature dependences of the refractive index and the thermal expansion coefficient. For a particular sample of fused silica, the absorption coefficient was determined to be $1.8 \pm 0.4 \times 10^{-5} \mathrm{~cm}^{-1}$. Obtaining this result requires a resolution in the optical path length better than $\pm 0.1 \mathrm{~nm}$.
\end{abstract}


There are many important optical systems where beam-quality requirements are sufficiently stringent that thermally-generated wavefront distortion becomes an issue. As an example, low-absorption fused silica is used for the transmissive optics in laser-based gravitational wave detectors (GWD).[1] These GWDs are designed to detect a strain of space-time on the order of $10^{-23}$; the strain appears as a phase difference in the light traveling in the two 4-km length arms of a Michelson interferometer. To enhance the signal-tonoise ratio, each interferometer arm is configured as an optical resonator; in addition, a power-recycling mirror located between the laser and beam splitter returns the light reflected from the interferometer back into it. Thus, both the beam splitter and the input mirrors of the arm resonators are exposed to continuous high transmitted power. Optical absorption in the substrates of these optics induces a spatial variation in their refractive indices, making them behave as lenses. This effect, known as thermal lensing, [2] changes the radius of curvature of the circulating light, reducing the power coupling into the interferometer and degrading the GWD's performance.[3]

Fused silica has quite low absorption at $1.06 \mu \mathrm{m}$, the wavelength of the Nd:YAG laser used in GWD's. Therefore, it is suitable for the material of the transmissive optics of these interferometers. However, because precise phase control of the circulating light is crucial for high performance of the GWD's, it is important to quantify the absorption coefficient of each optic individually so that the thermal effect can be calculated accurately. There are several known methods to estimate the absorption coefficient in lowabsorption materials. Boccara et al.[4] estimated the absorption of a fused silica by measuring the deflection that a probe beam suffers when it passes through the thermal lens caused by $1.06 \mu \mathrm{m}$ laser radiation. The key to successful measurement of the deflection using this method is an appropriate management of the spatial jitter (beam wandering) of the probe beam. Bunimovich et al.[5] employed laser calorimetry to estimate the absorption coefficient of fused silica fibers. They measured the temporal variation of the sample temperature caused by by turning on and off the heating laser and estimated the absorption coefficient from the thermal-rise and decay. Making use of the difference in the slope of the thermal rise, they separated the absorption coefficient of the surface from that of the bulk.[6]

In this paper we present a method for measuring absorption in highquality, low-absorption optical materials. We use a Shack-Hartmann wavefront detector (SHWD, manufactured by Wavefront Sciences, Inc.)[7] to measure the thermal lens due to high-power $(10 \mathrm{~W}, \mathrm{cw})$ laser radiation. [8] To cope 
with very low absorption, we have developed a method for reducing noise associated with laser intensity fluctuation and spatial jitter. By fitting the spatial profile of the measured thermal lens to a model for thermal deformation, we were able to measure an absorption coefficient of $1.8 \pm 0.4 \times 10^{-5} \mathrm{~cm}^{-1}$ in a $2.5-\mathrm{cm}$ long sample of fused silica. This method is suitable for applications where the overall temperature rise of a massive sample is negligibly small but the temperature gradient is enough to cause thermal lensing.

Fig. 1 illustrates our method. The fused silica sample (Suprasil, manufactured by Heraeus) used in this measurement was a cylinder $2.5 \mathrm{~cm}$ in diameter and $2.5 \mathrm{~cm}$ long. We exposed the sample to $10 \mathrm{~W}$ radiation from a cw Nd:YAG laser (Lightwave, model 220-1064-10000) operating in the TEM $_{00}$ mode (the "heating beam"), and probed the thermally induced optical path length change $\triangle O P L$ by a Helium-Neon laser (the "probe beam"). The heating beam and the probe beam propagated in opposite directions through the sample.

Fig. 2 illustrates the operation of the SHWD. The SHWD consists of a two-dimensional array of $20 \times 20$ lenslets mounted in front of a charge-coupled device (CCD). The diameter and the focal length of the lenslets in the SHWD used in this measurement are $0.25 \mathrm{~mm}$ and $25 \mathrm{~mm}$, respectively. Each lenslet focuses the part of the probe beam incident on it onto the CCD. The specific CCD element on which the focal spot sits depends on the angle with which the probe beam enters the lenslet. The position of the focal point can be estimated accurately by the centroid algorithm.[7] The SHWD reconstructs the wavefront by integrating the tilt over all the lenslets. Because each tilt represents $\triangle O P L$ over the diameter of the corresponding lenslet (Fig. 3), the reconstructed wavefront represents $\triangle O P L$ for the entire probe beam as a function of the lateral location $(r, \theta)$, and can therefore be used to determine the thermal lens. Here $(r, \theta)$ are the polar coordinates on the cross-sectional plane of the sample. $\triangle O P L(r, \theta)$ is then averaged with respect to $\theta$ and the spatial profile of the thermal lens is evaluated as a function of $r$.

When a Gaussian beam goes through an optic, a thermal lens is formed by the following mechanism. The radial intensity profile of the Gaussian beam induces a temperature gradient, causing spatial variation of both the refractive index and the physical length, on account of the temperature variations of these quantities. Assuming that the sample is much larger than the heating beam and that absorption is uniform in the sample, the steady-state, 
radial temperature distribution is given by $[2,8]$

$$
\Delta T(r)=\frac{P \gamma l}{4 \pi \kappa}\left[\sum_{i=0}^{\infty} \frac{(-1)^{i}\left(2 \frac{r^{2}}{w^{2}}\right)^{i}}{i i !}\right]
$$

where $\Delta T$ is the relative temperature at radius $r$ (referenced to the center of the Gaussian profile), $P$ is the laser power, $\gamma$ is the absorption coefficient, $l$ is the sample length along the beam path, and $\kappa$ is the thermal conductivity. Using an empirically derived approximation, the change in optical path difference $\triangle O P L$ as a function of the radius becomes

$$
\Delta O P L=0.07741 \frac{P \gamma l}{\kappa}\left(\frac{d n}{d T}+\alpha n\right)\left[\left(\frac{r}{w}\right)^{2}+0.4\left(\frac{r}{w}\right)\right]
$$

where $n$ is the index of refraction, $T$ is the temperature, $\alpha$ is the thermal expansion coefficient, and $w$ is the spot size of the heating beam. This approximation is applicable only for the case $r<w$.

For thermal lens measurements, the SHWD is usually used in the following way. First an image file is taken with the probe beam transmitted through the sample without exposing it to the heating beam. The positions on the CCD plane of the focal points from all the lenslets are determined by the centroid algorithm. These focal positions are called the reference centroids and the file containing the reference centroids is called the reference file. Next, the heating beam is superposed on the probe beam collinearly, keeping all the optics in the probe beam path unchanged.[8] The centroids (called the data centroids) are determined in the same way. Then by comparing data centroids and the reference centroids for the respective lenslets, the displacements of the focal positions are calculated. From these displacements the changes in the slope of the wavefront (called the tilts) are calculated for the respective lenslets.

In our case, some extra arrangements were needed to cope with the extremely small absorption, and corresponding small thermal lens. First, we optimized the heating beam size relative to the probe laser beam size. Because $\triangle O P L(r)$ varies as a function of $r / w$ where $w$ is the spot size of the heating beam [see Eq. (2)], $\triangle O P L /$ lenslet increases as the heating beam size decreases relative to the probe beam size. (In other words, as the heating beam size decreases relative to the lenslet size on the object plane, each lenslet sees a larger portion of the thermal lens.) Therefore, to enhance the 
tilt per lenslet, it is desirable to reduce the size of the heating beam relative to the probe beam. However, if the heating beam size is too small, the total number of lenslets involved in the whole thermal lens diameter becomes too small, resulting in considerably lower spatial resolution. Thus, there are optimum values for the sizes of both beams. We set the spot sizes of the heating beam and the probe beam at the sample location to be $0.7 \mathrm{~mm}$ and $2.5 \mathrm{~mm}$, respectively. We optimized these values empirically. The Gaussian probe beam was large enough to provide fairly uniform illumination over the entire pump beam.

Second, we collimated the probe laser so that its wavefront was as flat as possible, and adjusted the probe laser intensity as high as allowed by the linear range of the CCD. When the probe wavefront is flat, the reference centroid is located close to the center of the lenslet. In this way, the influence of lenslet-to-lenslet optical crosstalk[7] can be greatly reduced. Also, by increasing the intensity, the precision of intensity digitization to 256 (eight bit) gray levels is increased; hence, the accuracy of the numerical operation increases.

Third, to get good signal-to-noise ratio (SNR), we used averaging both of angle $\theta$ and of the results of several runs. In addition, we made measurement both with and without the heating beam on to allow for variations in the probe beam. With the size of the thermal lens in this measurement, the tilt caused by spatial jitter (beam wandering) of the probe laser is not negligible. Consequently, if the above-mentioned usual procedure is taken, the reconstructed wavefronts are different from run to run. To overcome this problem, we adopted the following procedure: (1) Make a reference file as usual. (2) Make $\mathrm{N}$ additional measurements without applying the heating beam. $\mathrm{N}$ is typically 10 but can be as large as necessary to get a satisfactory SNR. (3) Calculate the tilts for each of these $N$ measurements using the reference file made in (1). (4) Take an average of the resultant $\mathrm{N}$ data sets. (5) Reconstruct a wavefront from the average of the $\mathrm{N}$ data sets made in (4). (6) Evaluate the spatial profile $\triangle O P L(r)$ by averaging $\triangle O P L(r, \theta)$ with respect to $\theta$. (The resultant $\triangle O P L(r)$ is called the $0 \mathrm{~W}$ data.) The measured center of the probe beam is chosen to be the center of the polar coordinate system when this average is made. Then, (7) expose the sample to the $10 \mathrm{~W}$ heating beam, make $\mathrm{N}$ measurements, and repeat (3) through (6) using the same reference file as the $0 \mathrm{~W}$ data. (The resultant $\triangle O P L(r)$ is called the $10 \mathrm{~W}$ data.) Finally, (8) subtract the $0 \mathrm{~W}$ data from the $10 \mathrm{~W}$ data to evaluate the thermal lens as a function of $r$. In this work we used a modal wavefront 
reconstruction based on a second-order Cartesian polynomial[9] in step (5). When $\triangle O P L(r, \theta)$ is averaged over $\theta$ in step (6) we approximate the integration along the circumference at a constant $r$ by summation over square pixels having a finite width. Therefore, as $r$ decreases the quantization error becomes larger. In addition, the slope of $\triangle O P L(r)$ decreases as $1 / r$, making the extracted tilt more vulnerable to unavoidable noise such as electric noise. In the analysis below, we discard $\triangle O P L(r)$ data obtained in the range of $r / w<0.2$.

The jitter that the probe laser experiences during the acquisition of $\mathrm{N}$ data sets is reduced by this averaging, reducing the run-to-run variation in $\triangle O P L(r)$. The resultant averaged $0 \mathrm{~W}$ and $10 \mathrm{~W}$ data still contain the influence of the jitter that the probe laser suffers at the moment when the image for the reference file is taken. However, because both the $0 \mathrm{~W}$ and $10 \mathrm{~W}$ data are based on the same reference file, this influence is removed when the $0 \mathrm{~W}$ data is subtracted from the $10 \mathrm{~W}$ data in step (8).

Once the spatial profile of the wavefront is obtained, the absorption coefficient can be calculated by fitting the profile to Eq. (2). Provided that all the other constants are known, the absorption coefficient can be estimated by fitting the measured thermal lens profile to the theoretical profile calculated by Eq. (2) using the absorption coefficient $\gamma$ as the fitting parameter. We estimated the absorption coefficient in this fashion. Table 1 lists the numbers we used for this calculation.[10]

Fig. 4 shows an example of $10 \mathrm{~W}$ data as a function of $r / w$, together with the corresponding $0 \mathrm{~W}$ data. The deviation of the $0 \mathrm{~W}$ data from the horizontal axis (i.e. the line corresponding to $\triangle O P L(r)=0$ ) indicates the influence of the probe beam's jitter on the reference measurement. The increase in deviation towards the edge of the beam illustrates the larger influence of the jitter at the edge (because the wavefront tilts more near the edge).

We repeated these measurements five times. All the resultant $\triangle O P L(r)$ fall between the theoretical curves calculated from Eq. (2) for absorption coefficients of $1.2 \times 10^{-5} \mathrm{~cm}^{-1}$ and $2.2 \times 10^{-5} \mathrm{~cm}^{-1}$. Fig. 5 shows $\triangle O P L(r)$ averaged over all these data, where the two dotted lines are theoretical values corresponding to these limiting values of the absorption coefficient. After the averaging, the measured data best fits to a theoretical curve corresponding to an absorption coefficient of $1.64 \pm 0.32 \times 10^{-5} \mathrm{~cm}^{-1}$. To assess the level of possible thermal lensing by the dichroic mirror, we measured $\triangle O P L(r)$ without having a fused silica sample in the setup shown in Fig. 1. No $\triangle O P L(r)$ 
was observed, indicating that the thermal lensing effect in the dichroic mirror is negligible.

The $\triangle O P L(r)$ shown in Fig. 5 represents the change in the wavefront curvature observed at the image plane of the SHWD. In contrast, thermal lensing in the fused silica sample causes a change in the wavefront curvature at the location where the sample is placed. In general, when a lens is placed on the optical path of a Gaussian beam, the resultant change in the wavefront curvature depends on where you observe it, because the beam diverges as it propagates. In our setup, we needed to place the dichroic mirror between the sample and the SHWD (Fig. 1). Therefore, there is a finite distance between the observation point and the sample location, making the optical path length change observed by the SHWD not strictly the same as the optical path length change caused by the thermal lensing. Now we assess the correction associated with this fact.

To make this assessment simpler, we approximate the thermal lens by a spherical lens of focal length $f$, and place it in the optical path of a Gaussian beam. Although the thermal lens is not exactly a spherical lens, as indicated by Eq. (2), this approximation will be adequate for this assessment. We have calculated the overlap integral to estimate the intensity coupled into higher-order Gaussian modes by the non-spherical term of a thermal lens represented by Eq. (2). According to this calculation, the intensity in these modes for our observed $\triangle O P L(r)$ is less than $0.1 \%$.

Suppose now that the Gaussian beam has its waist at $z=0$, and that the lens and the SHWD are placed at $z=z_{f}$ and $z=z_{\text {obs }}$, respectively. By considering the propagation of Gaussian beams and using ray matrices,[11] the radius of curvature at $z=z_{o b s}$ of the probe beam, $R_{o b s}^{10 W}$, can be written as follows.

$$
R_{o b s}^{10 W}=\frac{\left[\left(\frac{1}{R_{f}}-\frac{1}{f}\right)\left(1+\Delta z\left(\frac{1}{R_{f}}-\frac{1}{f}\right)\right)+\Delta z\left(\frac{\lambda}{\pi w_{f}^{2}}\right)^{2}\right]^{2}+\left(\frac{\lambda}{\pi w_{f}^{2}}\right)^{2}}{\left[\left(\frac{1}{R_{f}}-\frac{1}{f}\right)^{2}+\left(\frac{\lambda}{\pi w_{f}^{2}}\right)^{2}\right]\left[\left(\frac{1}{R_{f}}-\frac{1}{f}\right)\left(1+\Delta z\left(\frac{1}{R_{f}}-\frac{1}{f}\right)\right)+\Delta z\left(\frac{\lambda}{\pi w_{f}^{2}}\right)^{2}\right]}
$$

where $R_{f}$ is the radius of curvature incident to the lens, $\lambda$ is the wavelength of the probe laser, $w_{f}$ is the spot size of the probe beam at the lens, $f$ represents the focal length of the thermal lens, and $\Delta z$ is the distance between the lens and observation point. When the heating beam is absent, the probe laser's 
curvature at $z=z_{o b s}$ is

$$
R_{o b s}^{0 W}=\frac{\left[\frac{1}{R_{f}}\left(1+\frac{\Delta z}{R_{f}}\right)+\Delta z\left(\frac{\lambda}{\pi w_{f}^{2}}\right)^{2}\right]^{2}+\left(\frac{\lambda}{\pi w_{f}^{2}}\right)^{2}}{\left[\left(\frac{1}{R_{f}}\right)^{2}+\left(\frac{\lambda}{\pi w_{f}^{2}}\right)^{2}\right]\left[\frac{1}{R_{f}}\left(1+\frac{\Delta z}{R_{f}}\right)+\Delta z\left(\frac{\lambda}{\pi w_{f}^{2}}\right)^{2}\right]}
$$

The optical path length change measured by the SHWD at $z=z_{o b s}, \triangle O P L_{o b s}$, is proportional to the difference between $1 / R_{o b s}^{0 W}$ and $1 / R_{o b s}^{10 W}$

$$
\triangle O P L_{o b s} \propto \frac{1}{f_{\text {eff }}}=\frac{1}{R_{o b s}^{0 W}}-\frac{1}{R_{o b s}^{10 W}}
$$

where $f_{\text {eff }}$ indicates the effective focal length evaluated at $z=z_{o b s}$ by Eq. (5). Then the question becomes how $f_{\text {eff }}$ is different from the true $f$. From Fig. 5 and the spot size of the heating beam at the thermal lens, $f_{\text {eff }}$ of this measurement can be estimated to be $670 \mathrm{~m}$. From an analysis of the reference file, we estimate that the probe laser has a waist of $0.348 \mathrm{~mm}$ at a position $4.62 \mathrm{~m}$ away from the fused silica sample (Fig. 1). From these numbers together with $\Delta z=0.22 \mathrm{~m}$, and using Eqs. (3)-(5), we can explore what value of $f$ gives the closest number to the measured $f_{\text {eff }}$ of $670 \mathrm{~m}$. When $f$ is $612 \mathrm{~m}, f_{\text {eff }}$ calculated by Eq. (5) is closest to the measured value of $670 \mathrm{~m}$, indicating that the focal length evaluated at $z=z_{\text {obs }}$ is $9.6 \%$ larger than the true focal length at $z=z_{f}$. In fact our calculation indicates that this error factor is fairly insensitive to the value of $f$, varying from 9.4-9.6\% while $f$ varies from $100-1000 \mathrm{~m}$. Therefore, it is reasonable to use the error factor of $9.6 \%$ for the above-mentioned absorption coefficient corresponding to the best-fit $\triangle O P L$, and estimate the corrected absorption coefficient to be $1.8 \pm 0.35 \times 10^{-5} \mathrm{~cm}^{-1}$. This absorption coefficient is about $20 \%$ higher than the value of $1.51 \times 10^{-5} \mathrm{~cm}^{-1}$ that Boccara et al.[4] estimated by beam deflection technique on samples of the same material. It is two orders of magnitude smaller than the absorption coefficient estimated by Bunimovich et al.[5] for a fused silica fiber.

Finally we briefly discuss the meaning of the $\triangle O P L$ in these measurements. In Figs. 4 and 5, the resolution of this measurement appears to be better than $0.1 \mathrm{~nm}$, which corresponds to a wavefront slope of $0.1 \mathrm{~nm} / 250 \mu \mathrm{m}=4 \times$ $10^{-7} \mathrm{rad}$. This value is an order of magnitude better than the resolution estimated for a similar wavefront measurement using the same type of SHWD[7] where the authors claim that the limiting factors for the resolution are sensor noise and lenslet-to-lenslet optical crosstalk. We believe that in our case the 
above-mentioned optimization of the wavefront and intensity of the probe laser reduces the optical crosstalk greatly. According to a numerical calculation made by Armstrong,[12] the wavefront slope of $4 \times 10^{-7}$ (rad) corresponds to a theoretical RMS centroid error due to pure electrical amplitude noise (gain variation in pixels) when an error of $0.1 \%$ of the maximum amplitude is assumed. It seems that the averaging procedure we employed contributes to reduction of the electrical noise.

Unlike the calorimetric approach employed by Bunimovich[6], our method cannot separate the surface absorption from the bulk absorption in a single measurement. However, as Eq. (2) indicates, the contribution of surface absorption to the measured $\triangle O P L$ is proportional to the product of the absorption coefficient and the thickness of the surface layer. Therefore, unless the absorption coefficient of the surface layer is much larger than the bulk or the bulk length is comparable to the surface thickness surface absorption givea a much smaller thermal lens than bulk absorption. In the case of the fused silica transmissive optics used in the above-mentioned GWD's, the surface absorption is two orders of magnitude lower than the bulk absorption [13]. If it were necessary to separate the surface absorption, we can prepare two samples having the same surface conditions and different bulk length. Then the bulk absorption can be estimated from the difference in $\triangle O P L$ of the two samples.

In summary, we have demonstrated a measurement of very low absorption in fused silica using a Shack-Hartmann wavefront detector to measure the properties of a thermal lens introduced by a $10 \mathrm{~W} 1.06 \mu \mathrm{m}$ wavelength laser. By adjusting the beam shape and intensity of the probe laser appropriately and by averaging the acquired image data, we have been able to measure $\triangle O P L$ on the order of $10^{-10} \mathrm{~m}$. From the measured $\triangle O P L$ we have estimated the absorption coefficient of the fused silica sample to be $1.8 \pm 0.4 \times 10^{-5} \mathrm{~cm}^{-1}$.

\section{Acknowledgments}

We thank Q. Shu for his helpful discussion on the data correction associated with the divergence of the probe beam. This work was supported by the National Science Foundation through grants PHY-0070854 and PHY9900786. 


\section{References}

[1] A. Abramovici, W. Althouse, R. W. P. Drever, Y. Gursel, S. Kawamura, F. J. Raab, D. Shoemaker, L. Sievers, R. E. Spero, K. Thorne, R. E. Vogt, R. Weiss, S. E. Whitcomb, and M. E. Zucker, "The Laser Interferometer Gravitational-wave Observatory", Science 256, 325 -333 (1992).

[2] K. Strain, K. Danzmann, J. Mizuno, P. Nelson, A. Rüdiger, R. Schilling, W. Winkler, "Thermal lensing in recycling interferometric gravitational wave detectors", Phys. Lett. A. 194, 124 - 132 (1994).

[3] D. E. McClelland, J. B. Camp, J. Mason, W. Kells, and S. E. Whitcomb, "Arm cavity resonant sideband control for laser interferometric gravitational wave detectors", Opt. Lett., 24, 1014 - 1016 (1999).

[4] Claude Boccara, Laboratoire d'Optique Physique, Paris, France, private communication (1998).

[5] D. Bunimovich, E. Belotserkovsky, L. Nagli and A. Katzir, "Measurements of absorption coefficients using noncontact fiber-optic laser calorimetry", Appl. Opt. 34, 743 - 745 (1995).

[6] D. Bunimovich, L. Nagli and A. Katzir, "Absorption measurements of mixed silver halide crystals and fibers by laser calorimetry", Appl. Opt. 33, 117 - 119 (1994)

[7] D. R. Neal, D. J. Armstrong and W. T. Turner, "Wavefront sensors for control and processing monitoring in optics manufacture", in Laser as tool for manufacturing II, L. R. Migliore and R. D. Schaeffer Eds., SPIE 2993, 211-220 (1997).

[8] J. D. Mansell, J. Hennawi and E. K. Gustafson, "Evaluating the effect of transmissive optic thermal lensing on laser beam quality with a ShackHartmann wave-front sensor", Appl. Opt. LP, 40, 366-374 (2001).

[9] Wavefront Science Inc, CLAS2D Operation Manual, Rev. 1.50a, p.69 (1999)

[10] Heraeus, Quartz Glass for Optics, Optical Properties, 1994 edition. 
[11] See for example, A. Yariv, Introduction to Optical Electronics, (Holt, Rinehart and Winston, New York, 1976), Chaps. 2 and 3.

[12] Darren Armstrong, WaveFront Sciences, Inc. Albuquerque, NM, private communication (1998).

[13] R. Beausoleil, Stanford University, Stanford, CA, private communication (2000). 
Table 1: Numbers used to estimate the absorption coefficient.

\begin{tabular}{ccccc}
\hline$\frac{d n}{d T}\left(\frac{1}{K}\right)$ & $\alpha\left(\frac{1}{K}\right)$ & $n$ & $l(\mathrm{~cm})$ & $\kappa\left(\frac{\mathrm{W}}{\mathrm{mK}}\right)$ \\
\hline $9.8 \times 10^{-6}$ & $5.1 \times 10^{-7}$ & 1.45 & 2.5 & 1.38 \\
\hline
\end{tabular}

Figure 1: Experimental setup. Dichroic mirrors are highly reflective at 1.06 $\mu \mathrm{m}$ and partially transmissive at $633 \mathrm{~nm} . z=0$ is the waist location of the probe beam. $z=z_{f}=4.62 \mathrm{~m}$, and $z=z_{o b s}=4.84 \mathrm{~m}$ denote the locations of the sample and the image plane of the SHWD, respectively.

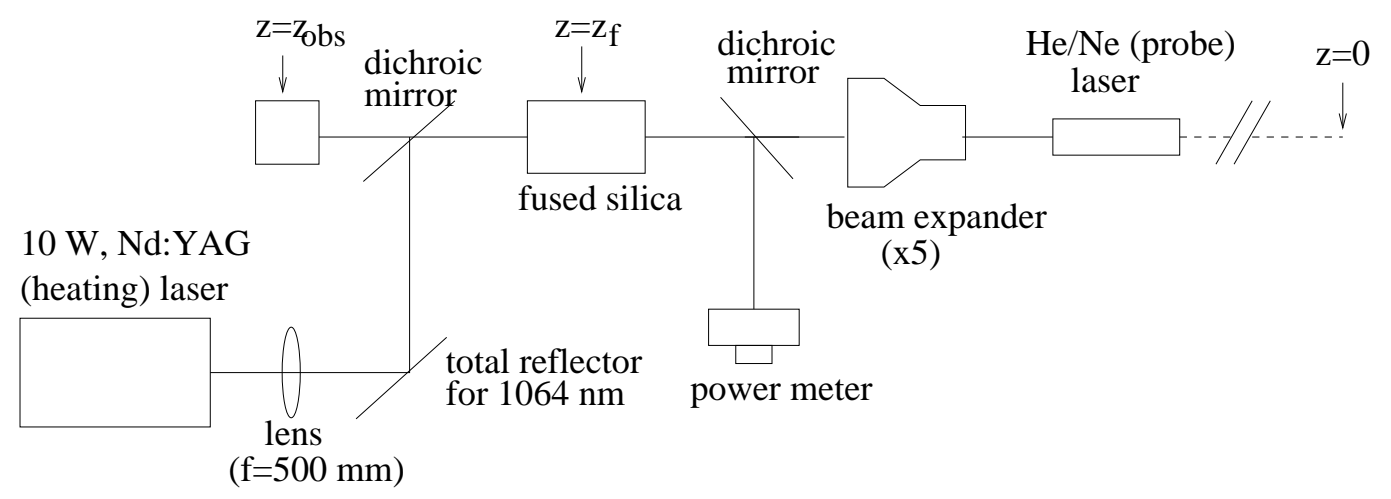


Figure 2: Schematic view of lenslet and detector arrays in the SHWD.

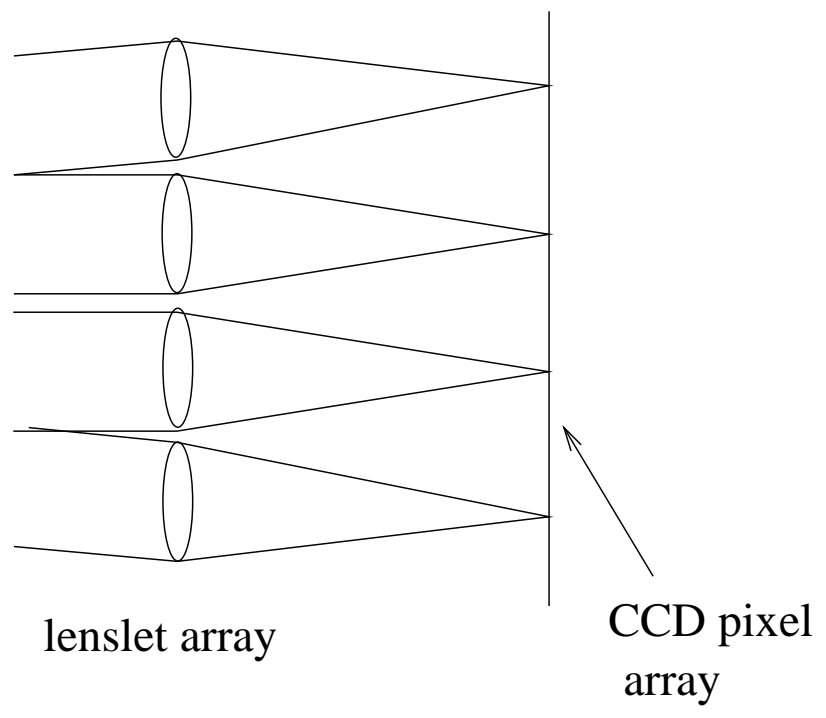


Figure 3: Schematic view of the relationship between the displacement of focal point on the detector plane $(\delta)$ and the change in the wavelength slope. The dashed line represents the reference wavefront and the solid line represents the data wavefront.

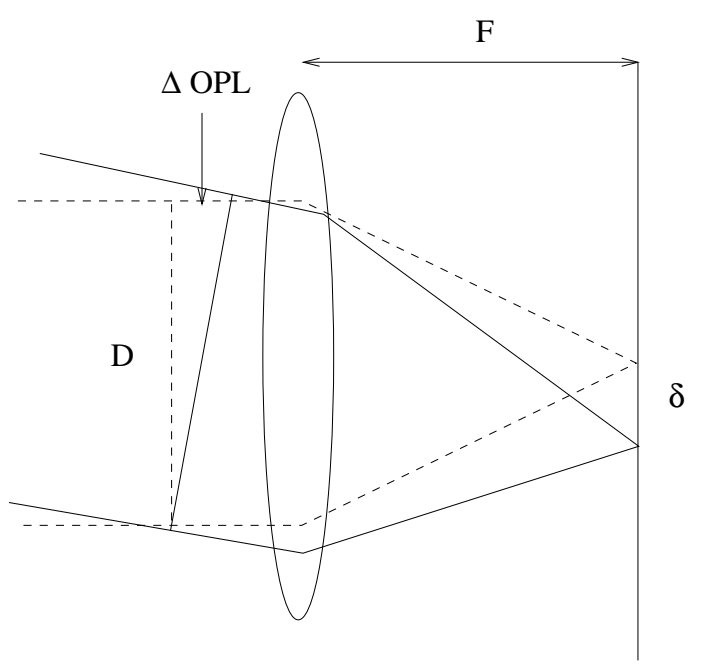

$$
\begin{aligned}
\Delta \mathrm{OPL} / \mathrm{D}=\delta / \mathrm{F} \\
\mathrm{D}: \text { lenslet diameter } \\
\mathrm{F}: \text { focal length } \\
\delta: \text { focus displacement }
\end{aligned}
$$


Figure 4: Sample $10 \mathrm{~W}$ and $0 \mathrm{~W}$ data.

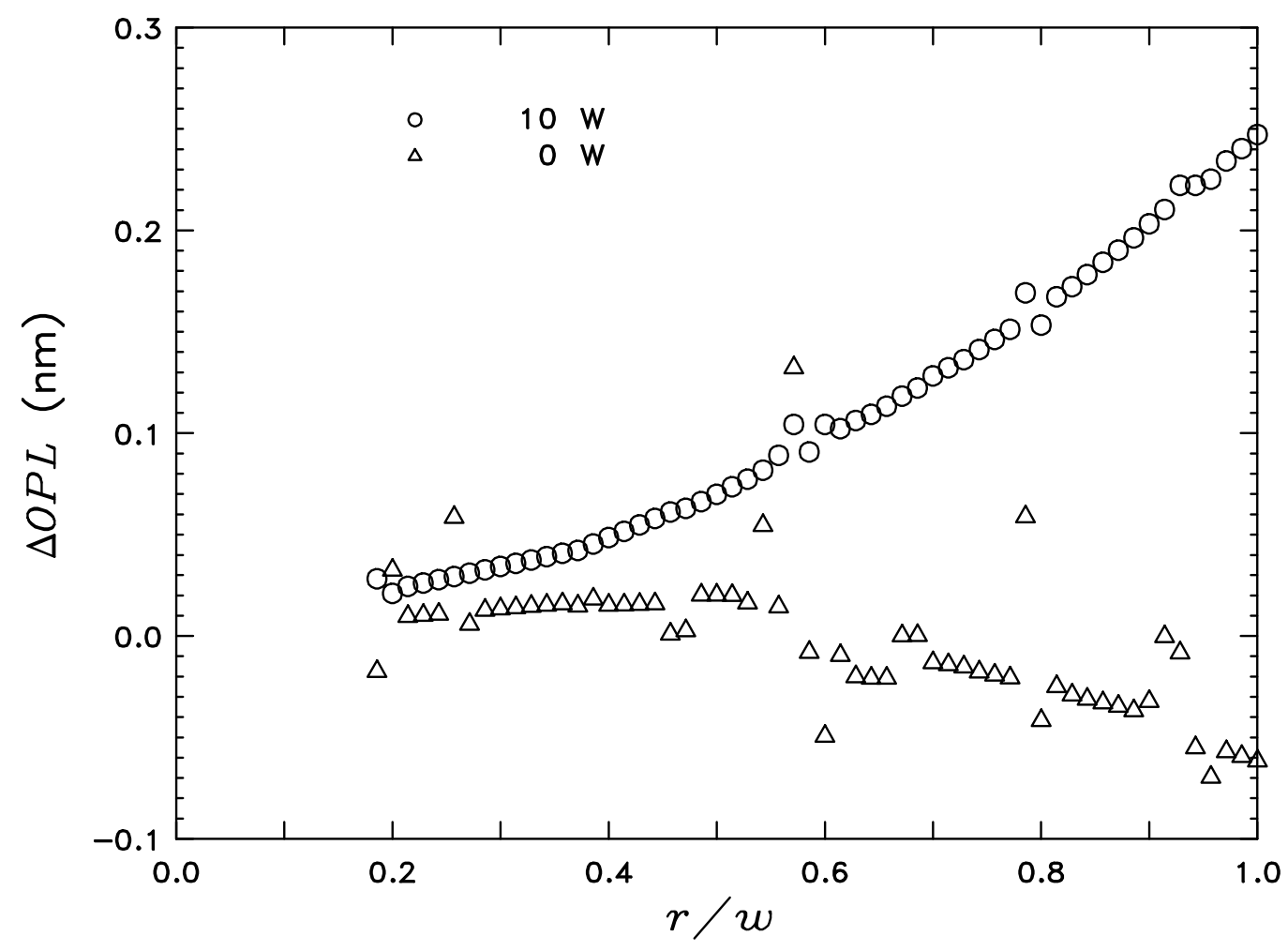


Figure 5: The points are the averaged wavefront at $10 \mathrm{~W}$. The lower dotted line shows the calculated wavefront for an absorption coefficient of $1.2 \times$ $10^{-5} \mathrm{~cm}^{-1}$; the upper dotted line is for $2.2 \times 10^{-5} \mathrm{~cm}^{-1}$.

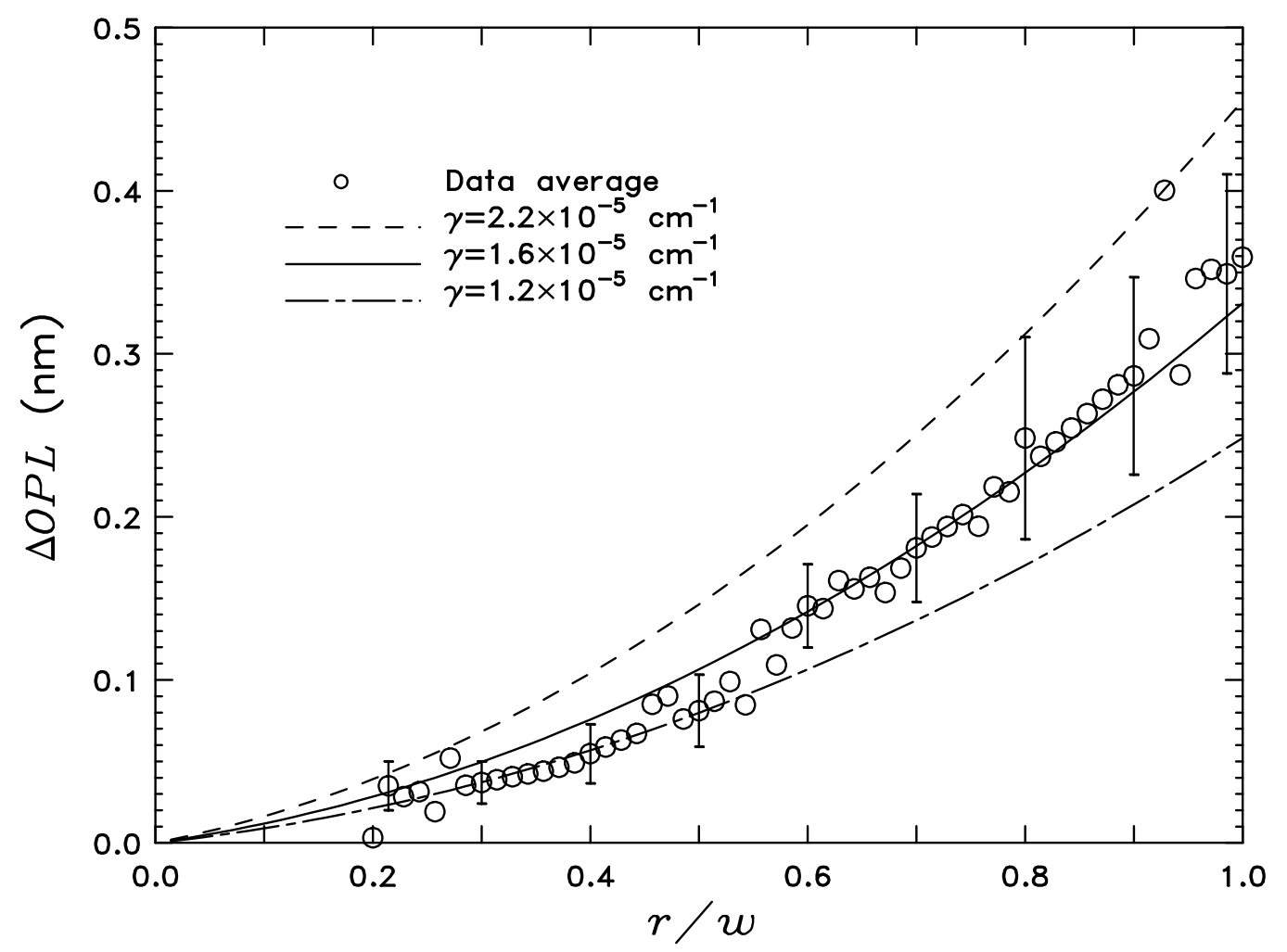

